\title{
Network System: The Integrated Picture Archiving and Communication System With the Hospital Information System
}

\author{
Isao Takeda and Toshihiko Kudo
}

\begin{abstract}
We describe the outline of Hokkaido University picture archiving and communication system (HU-PACS) and its network functions. HU-PACS processes 0.5 Gbytes of images daily through 10 acquisition devices. Functional integration of hospital information system (HIS) and PACS has been implemented. The HU-PACS can access common data base with HIS. Multi-image database systems provide fast throughput of image retrieval.

The system is composed of 4 modules: (1) 2 image data-base systems (IDS), (2) 1 image management system (IMS), (3) image display terminals (IDT), and (4) 8 image acquisition nodes interfaced to 10 modalities and 1 film scanner. These 4 modules are connected by branch and loop type local area networks (LAN). HU-PACS has functions other than basic PACS functions. Images in optical disk library (ODL) are loaded onto magnetic disks (MD) in advance according to patient booking information, the images expected to be viewed are transferred to the local storage automatically, and the desired images can be pre-downloaded into local storage by instruction through HIS terminals.
\end{abstract}

Copyright (C) 1991 by W.B. Saunders Company

KEY WORDS: PACS, HIS, RIS, network.

W ITH THE SPREAD of imaging systems and rapid progress in computer technologies, many hospitals are now planning to implement a picture archiving and communications system (PACS). In some of them, this new system has been already put in operation. In such a situation, communication networks play a very important role in making the most of PACS in actual clinical use. For this purpose, large-scale and high-speed networks must be configured to receive a great deal of data from the imaging systems. In constructing an actual PACS, attention should be paid to the performances of transmission lines prepared for it, as well as to the problems inevitably arising in it.

\section{NETWORK IN PACS}

\section{PACS Components}

In general, PACS is configured with components such as image acquisition nodes, image ar-

From the Medical Information Systems Division, NEC Corporation, and the Division of Medical Informatics, Hokkaido University Hospital, Sapporo, Japan.

Address reprint requests to Isao Takeda, Medical Information Systems Division, NEC Corporation

Copyright (C) 1991 by W.B. Saunders Company

0897-1889/91/0404-0113303.00/0 chiving equipment, image display/print terminals, and a transmission channel.

An image acquisition node is composed of two modules, one for interfaces of the images from computed tomography (CT), magnetic resonance imaging (MRI), computed radiography (CR), and a film scanner used to digitize conventional films and the other for the network to transfer the digitize image to archiving hosts.

The image archiving host uses optical disks as long-term storage. In midscale or larger systems, jukebox type devices that hold more than one optical disk and magnetic disks are used as highspeed short-term storage at the same time. Some transmission lines may be connected with image acquisition nodes and image terminals.

An image display terminal (IDT) has functions for retrieving and receiving images stored in archiving hosts to display them on its high-resolution display units. It has a magnetic disk that is used to store images for the short term. It also has various image manipulation functions that support clinical reading.

These components are organically integrated into one system through transmission lines such as local area network (LAN) or wide area network (WAN). By constructing a network in which each component can fulfill its functions, PACS is intended to offer a system to transfer at high speed and to display the required image at a required time at the required place, together with associated information.

\section{Function Required for Networks}

In a PACS network, a great amount of data must be effectively handled at high speed. Because simple connection of components on a highspeed line might not cope well with peak load, a very large scale and high-speed transmission line must be constructed, which causes economic problems. Therefore, PACS networks should be so configured that each of its components such as image input nodes, archiving hosts, and IDT may fulfill their functions to the utmost.

For PACS networks, the following functions must be realized: (1) More effective transfer of 
image input/retrieval, including improvement in throughput of transmission line and sharing a load; (2) more effective connection with external systems, including standardized interface; and (3) intelligent connection between (hospital information system) (HIS) and radiology information system (RIS)

a. Unified information management

b. Avoidance of duplicated entry of information into the two systems

c. Maintain the integrity of the databases

PACS NETWORK IN HU

\section{Fundamental Concepts for HU-PACS}

In HU Hospital, about 400,000 films are generated yearly. About 3G-bytes of these images are now handled daily by the PACS in this hospital. When these images are compressed to one third to one tenth of their original size, the amount of data to be treated is about 0.5 Gbytes. Moreover, almost all of the image data is generated for 2 concentrated hours in the morning, so that the load of the whole system reaches its peak at this time.

Under these conditions, a PACS that can be clinically used to offer high-quality and highspeed services has been designed and configured from the viewpoints of reliability and high effectiveness. Above all, to improve system throughput, this network is constructed on the basis of concepts which consist of sharing a load and preparing the required images beforehand at the required place (terminal).

\section{Configuration of HU-PACS}

As shown in Fig 1, the HU-PACS is configured with 1 image management system (IMS), 2 image data base systems (IDS), 8 digital data input terminals (DIT), 1 film input terminal (FIT), 4 image workstations (IWS), 16 IDT, 9 branch topology LANs (B4680), and 1 optical fiber loop LAN integrating these branch LANs.

Each component fulfills its functions as follows.

\section{Image Management System}

The hospital information system (HIS), which controls and manages all information in the hospital, has been implemented at HU Hospital. In this HIS, various kinds of information generated in the hospital are stored as data base on a host multiprocessor NEC ACOS 610 computer through 350 workstations. It is essential to make effective use of information in the HIS data base in constructing a practical PACS, so that control and management over PACS are assured by the host data base in HIS (Fig 2). One of the most important functions of the PACS is management of the image directory stored in two IDS. In the PACS, this module of the HIS is called IMS.

\section{Image Data Base System}

The HU-PACS has two IDS, each composed of a NEC super-minicomputer MS4120A. Each IDS has 16 Mbytes of RAM, 2.4 Gbytes of magnetic disk units, three ports of branch LAN $\mathrm{I} / \mathrm{F}$, and an optical disk library (ODL) equipped with two drives and 48 media slots. Each slot can hold a two-sided 2 Gbytes optical disk, so that each IDS has a 96 Gbytes long-term storage.

Each IDS functions as an image archiving host. It supports the various functions of PACS through the instructions from the IMS. At the IDS, image data is divided into image data and its demographic data when input into the system. The former data are saved in the ODL while the latter information is sent to the IMS, creating in this way an image directory.

\section{Digital Data Input Terminals}

A DIT is linked with each modality through a parallel interface, and uses its own compression/ extension hardware unit. The images captured from the imaging system are compressed into one third to one tenth of their original size and are transformed into the internal standard format before they are transferred into the IDS through the LAN.

\section{Image Displaying Terminals}

A local magnetic disk with a capacity corresponding to the amount of image data to be treated at each IDT is implemented as high-speed local storage. These IDT allow automatic receipt of images addressed to the terminal independent of operator's manipulation.

Image workstation. An IWS has the following components; 2 sets of high-resolution 20-inch 256 gray level displays, each having 1037 raster lines and a matrix of $960 \times 960$, a 14-inch CRT for image information retrieval, image memory, main memory, a high-speed image processing unit, a compression/reconstruction processing unit, a 160 Mbyte magnetic disk, a 32 bit CPU, 


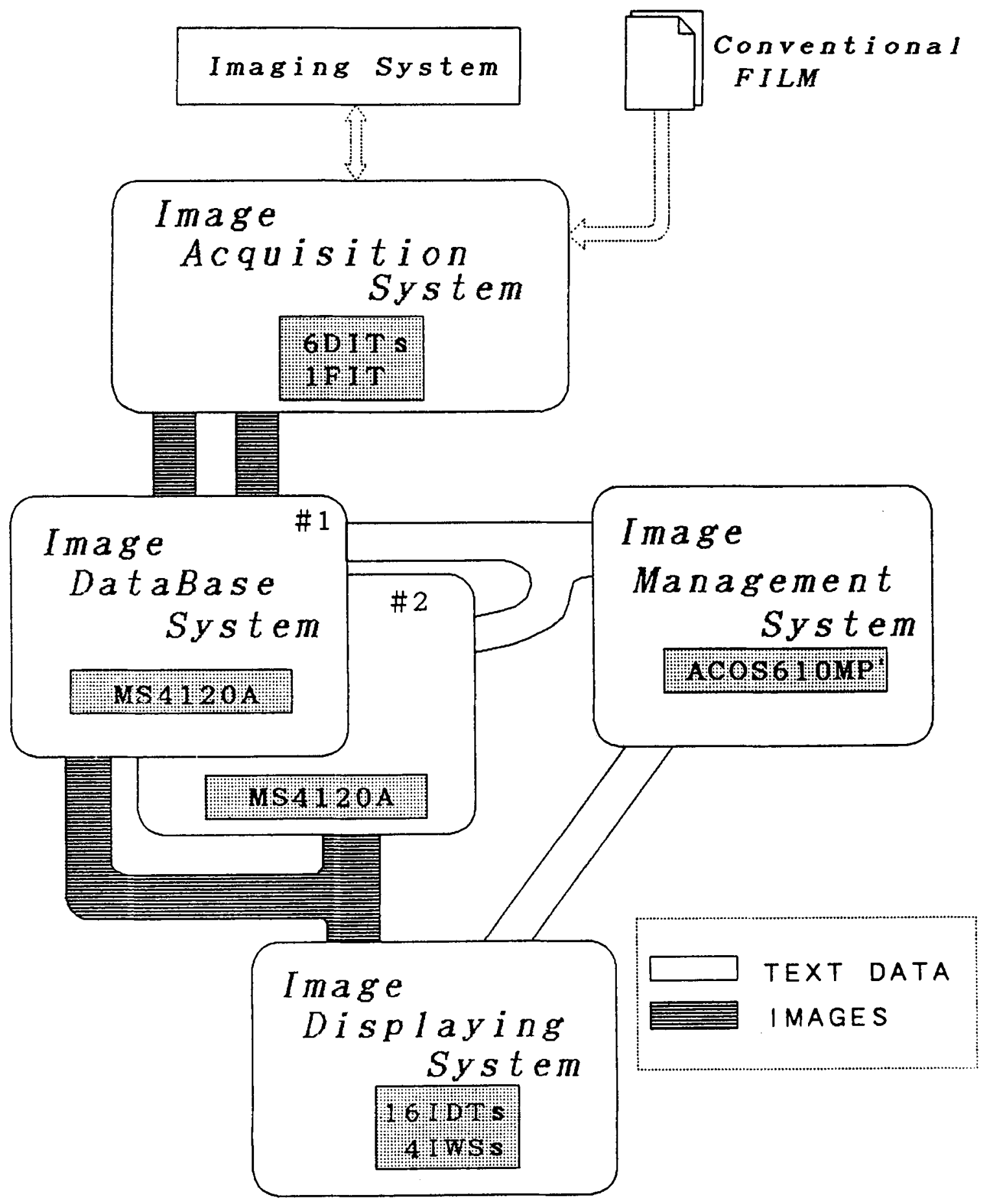

Fig 1. HU-PACS components.

and branch LAN adapter. The IWS is mainly for radiologists and has many image processing functions. We have installed 4 IWS in the radiology department.

Image display terminal. IDT has the following composition; a high-resolution 20-inch, 256 gray level, flicker-free screen with 1066 raster lines and a matrix of $1536 \times 1024$, image memory, main memory, a 80 Mbyte magnetic disk, an image processing unit, a compression/reconstruction unit, a 32 bit CPU, and branch LAN adapter. The IDT is used as a viewing station that can 
Fig 2. Relation of IMS module and HIS.

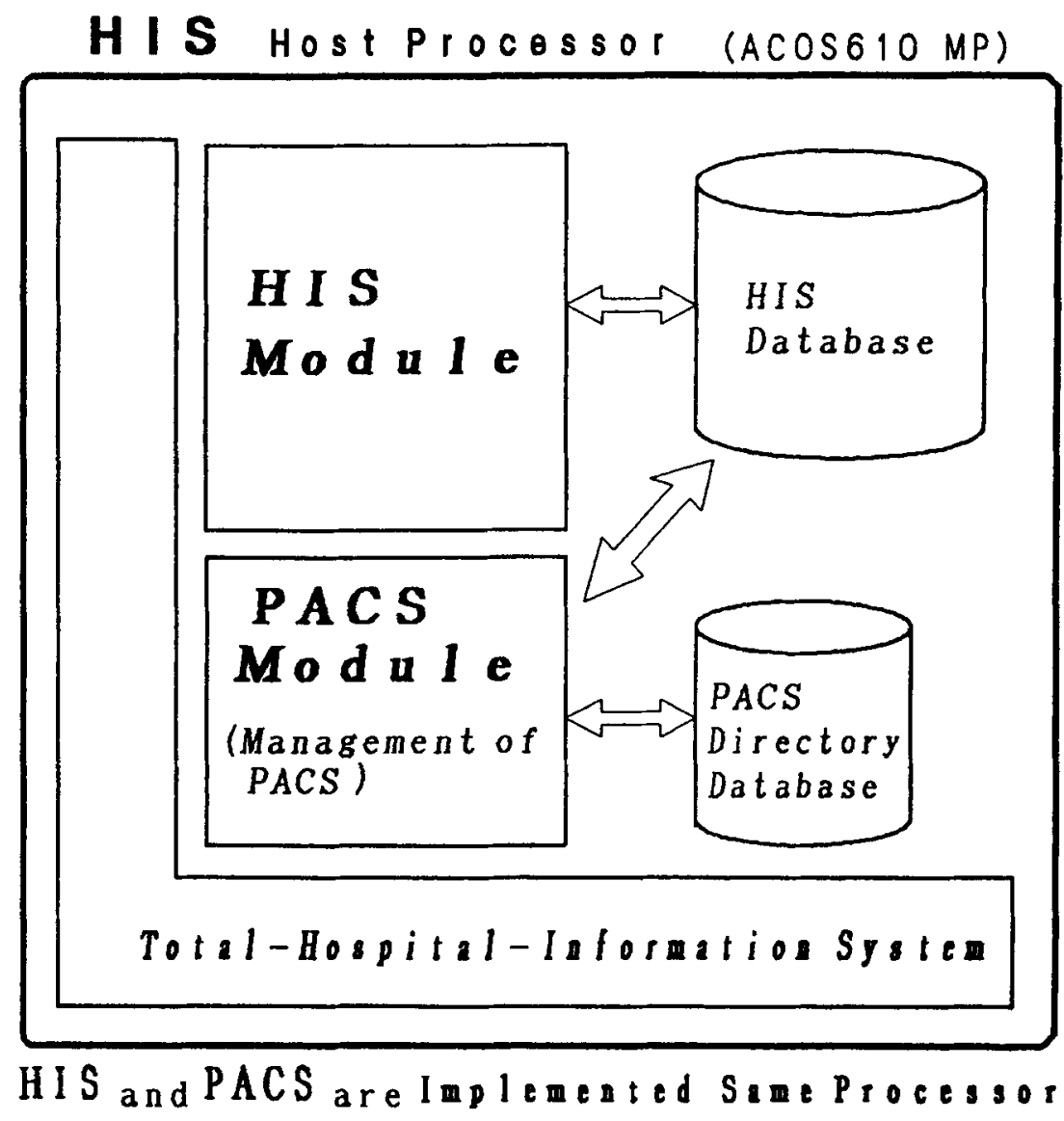

easily access images. Sixteen IDT sets are distributed throughout the orthopedic and general outpatient clinics.

\section{Image Transmission Line}

HU-PACS uses two modules of LAN, one for image acquisition section and the other for image reference section, so that the load generated by the image acquisition section may be separated from that of the entire PACS (Fig 3).

The network topology used in HU-PACS is a combination of loop and branch. In image reference LAN, an optical loop LAN is implemented as main LAN. Each image terminal, IMS and IDSs are connected together through nine branch LANs delivered from nodes on loop LAN.

Optical loop LAN: Loop 6770H. Loop6770H is an optical fiber cable LAN with a signaling transmission speed of $100 \mathrm{Mbps}$. One loop can make a connection with maximum 126 nodes (loop interface unit; LIU) each of which can connect with two branch LANs, as described later.
In HU Hospital, two sets of Loop6770H are installed, one used for more than 350 workstations in HIS and the other for PACS. The two sets of loop LAN are connected with each other through the IMS (ACOS610).

High speed and high reliability must be assured because these LAN function as an artery through which all the digitized information circulates. Each loop LAN has its own loop supervising unit (SVU), which can supervise data links and collect/control the operating information. It can assure high reliability by dual optical data highway and by offering bypass function, replacement bypass function, and loopback function.

Branch LAN: Branch 4680 . Branch 4680 is a 10 Mbps branch topology LAN using coaxial cable and adopting CSMA/CD method in accordance with IEEE802.3 as a standard Ethernet type LAN. The HU-PACS has more than one branch LAN for receiving and sending concentrated image data from the image data base, thus transferring a large amount of data effectively. In its reference section, nine Branch 4680 are 


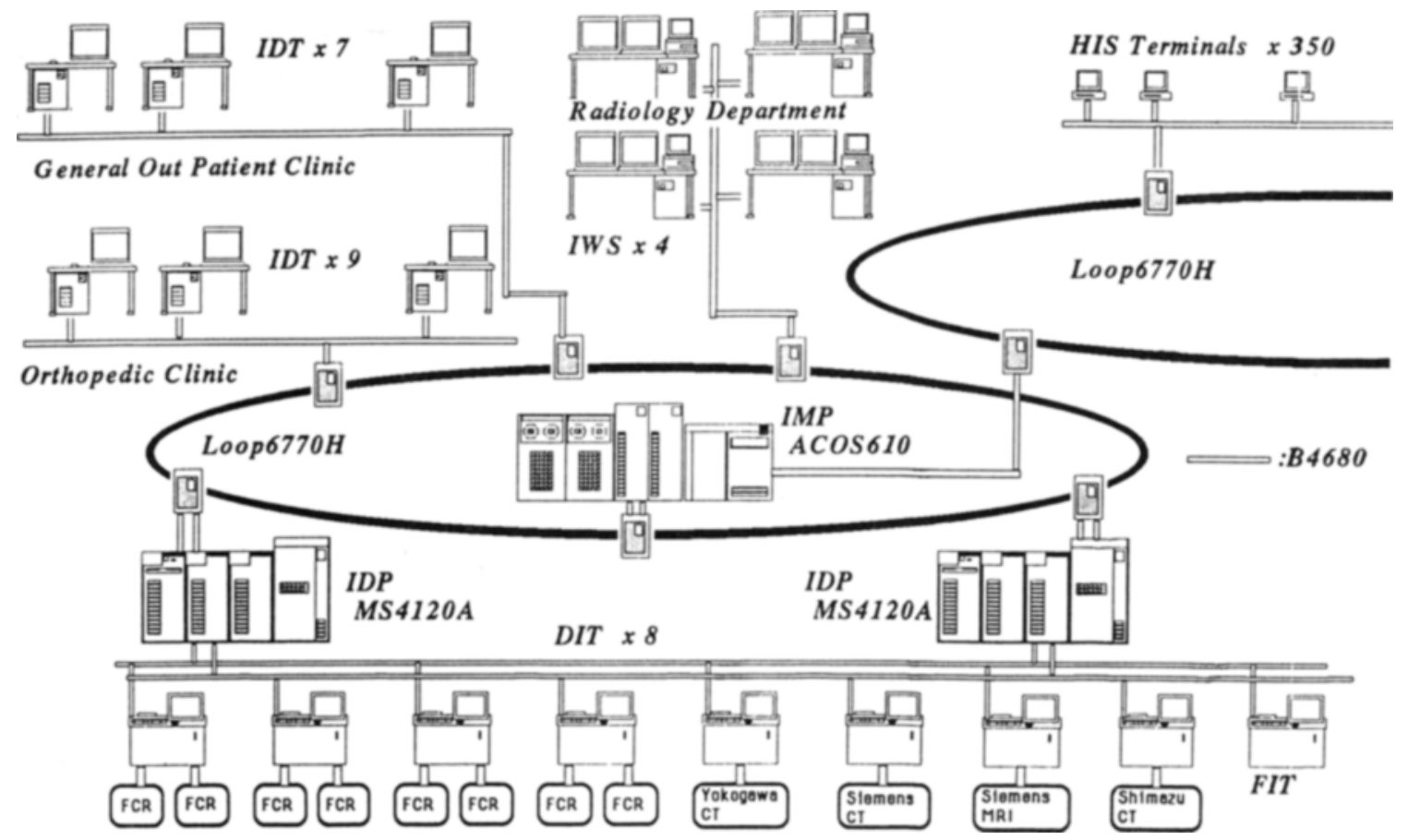

Fig 3. HU-PACS overview.

branched from LIU and connected with each node.

$W A N$. Public telephone lines and $9600 \mathrm{bps}$ modems are used to transmit images to other facilities.

\section{Network's Functions}

The HU-PACS is so linked that each of its components can fulfill its function to the utmost. An intelligent integration of HIS into PACS allows the following PACS functions to be implemented:

\section{Unified Management of Image Information}

Under actual conditions, all the image information stored on two IDSs is integrated by an IMS. At image terminals, retrievals such as the location of images and the history of examinations can be made by referring to the IMS data base. It allows implementation of more than one IDS. In the future, modality input and image terminals can be easily added merely by extending or enlarging an IDS according to the amount of data to be treated.

\section{Common Data Base Between HIS and PACS}

The IMS shares fundamental information data base on patients with HIS. This avoids duplicate data base and decreases the probability of data entry errors, so that images can be confidently registered into PACS merely by obtaining the required minimum information such as patient identification (ID) number from CT or MRI console.

As shown in Fig 4, as to FCR generating the largest amount of data, magnetic stripe cards that are used to take examinations are created at the HIS workstation as one of the reception services by radiology department. The information, such as patient's name, ID, section, sex, age, and the key number for image capture are encoded into the magnetic stripe cards. When FCR examinations are taken, the information on these cards is read and the data are transferred to the PACS with the images. In this way, the images can be stored at PACS with assurance.

\section{Automatic Delivery of Images}

Figure 5 shows how stored images change media. New images are permanently stored in the ODL and are also stored temporarily for about 1 week on the magnetic disks of IDS for rapid retrieval. Therefore, when a required image does not exist on local storage of an IDT, it can be transferred from the archive to high-speed magnetic disks on the archiving host. 


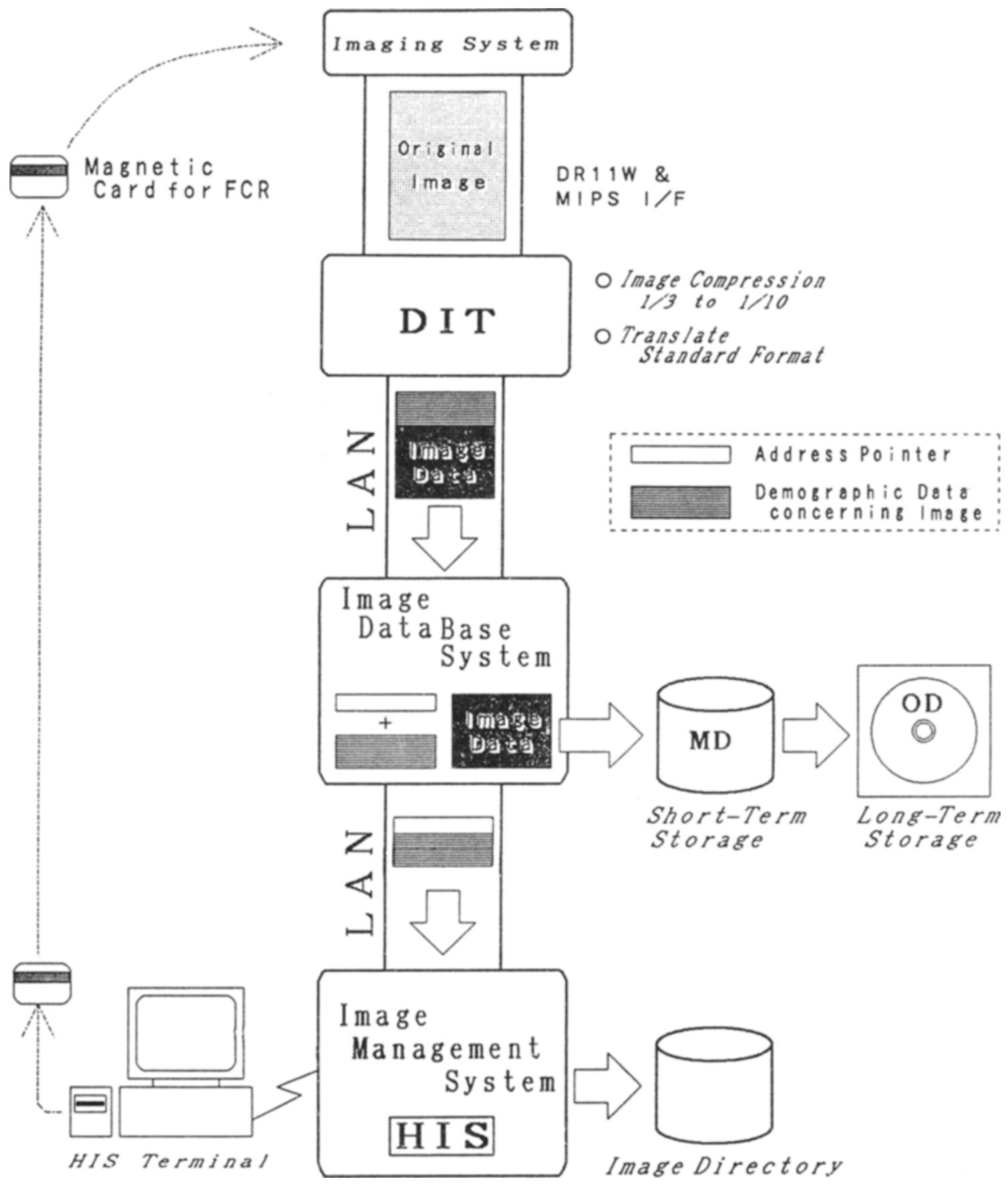

Fig 4. Image acquisition system.

On the other hand, accessing the ODL each time is not effective because it increases the time needed for image retrieval. In many cases, an image taken more than 1 week earlier is required as a reference image for a newly taken image. In HU Hospital, patients must make a reservation to receive treat- ments for the second time and for subsequent treatments. For this purpose, the information on patient's booking is registered by clinicians at HIS terminals installed at each outpatient clinic.

On the basis of this booking information, the IMS retrieves the image directory data base to 


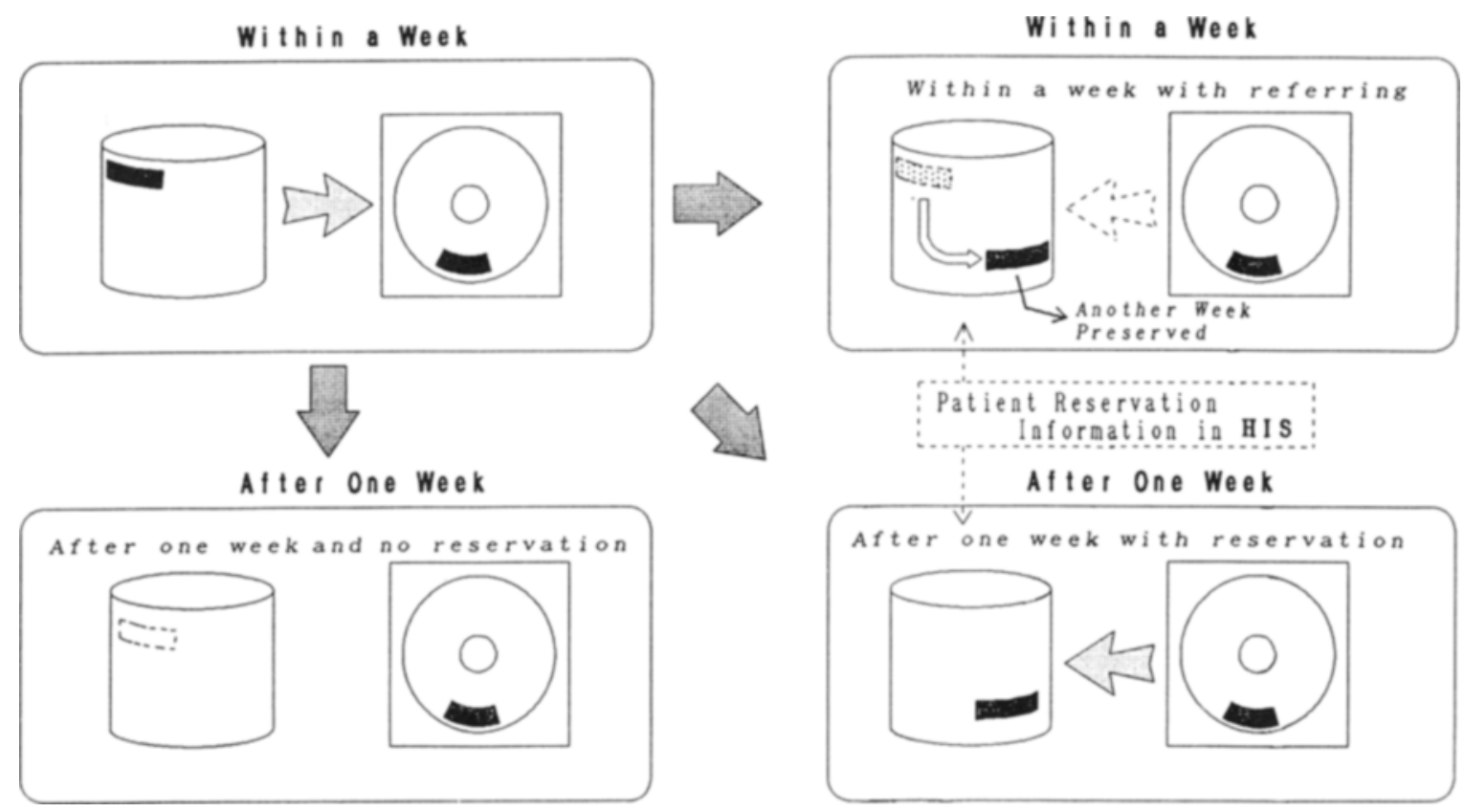

Fig 5. Relation of short-term and long-term storage.

obtain the address pointers of the patient's images. The IMS sends a delivery request to the IDS to restore all the patient's stored images from the ODL to magnetic disks during the night before the day of the visit. In this way, the time required to retrieve their images is remarkably shortened because images of each patient exists on magnetic disks on the day of the hospital visit.

\section{Preliminary Transfer of Images}

By using a HIS terminal, clinicians at each clinical department can reserve and indicate the terminals to which the images taken that day are to be sent (Fig 6). Once taken, "images on the day" are immediately stored on IDS through DIT while the image directory is updated on IMS. Then, if information on "reservation/instruction of addressed image" exists, the images are automatically transferred and stored on the given IDT. In addition to these images on the day, "reference images," which also exist on IDS magnetic disks can be transferred beforehand as well. With no special instruction, all images in the radiology department are automatically transferred and stored in local storage before reading.

\section{More Effective Image Retrieval}

As shown in Fig 7, the images are managed in a hierarchical way in the image directory data base on IMS, so that a retrieval from image terminals can be made effectively in accordance with this hierarchy. In general, the images are often actively retrieved from image terminals on the basis of "examination series." In this case, the images already transferred to terminals can be displayed simultaneously even during the transfer of more than one image in an examination series.

\section{Reference to Radiology Report}

Diagnostic reports produced by radiologists are stored in the HIS data base as very important information on the PACS so that they can be retrieved at any of the 350 workstations within the HIS. At image terminals located in services other than the radiology department, clinicians cannot review images before the reports of those images are stored in the HIS database.

\section{Teleradiology}

By use of public telephone lines, images can be transmitted between Nakashibetu Municipal Hospital located $400 \mathrm{~km}$ from HU and HU Hos- 


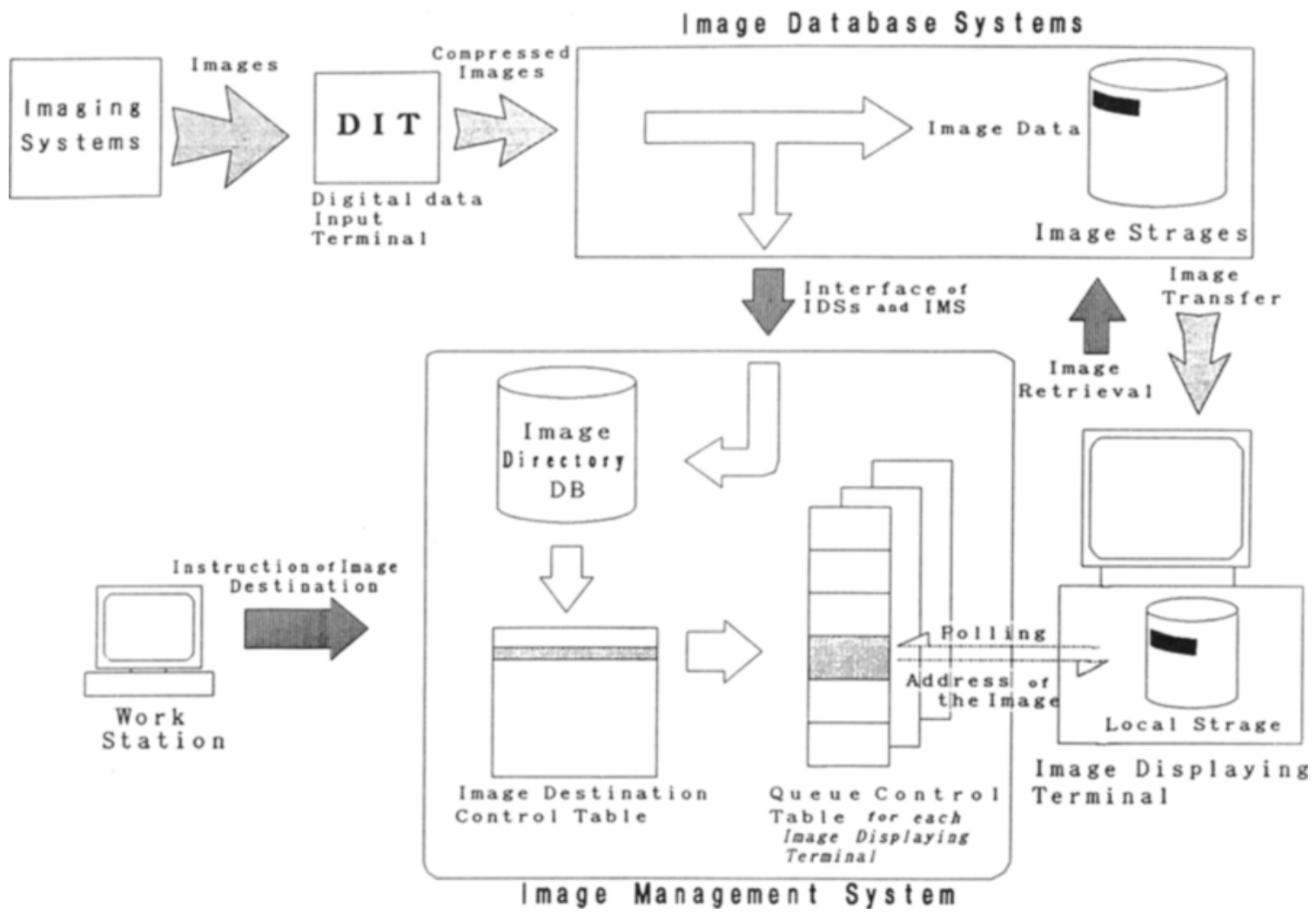

Fig 6. Reservation and instruction for prefetching.

pital. From Nakashibetu, CT images are input through an input and transmission terminal named Medifile 1000 and then are compressed into one fifth to one tenth their original size before being transferred through 9600 bps modems to HU-PACS. The images transferred are received and stored in the local storage at PACS input terminal. The radiologist can then read these images on high-resolution CRT and send reports on the images to Nakashibetu.

\section{PROBLEM TO BE RESOLVED}

Using HU Hospital as an example, we have described network functions required for PACS. With further spread of PACSs in future, these new systems will be connected with various kinds of imaging systems so that they will have to resolve problems arising from those interfaces. Such problems are likely to be resolved by imaging equipment compatible with MIPS digital image communication standards, which is a subset of ACR/NEMA. However, improvements should be made in interfaces to capture images from imaging systems to PACS as well as in bidirectional interfaces to link the information input by clinical department on examination order through HIS, RIS, and PACS. In this way, images stored in PACS may be better used.

It is likely that PACS will be installed in other institutions, making possible mutual exchange of digitalized image information between facilities. For this purpose, flexible media and WAN generally will be used. With regard to the former media, 5-inch magnet optical disks are positively standardized to make them usable for this purpose while image save and carrying (ISAC) systems based on them are being investigated. For the latter networks, ISDN is now considered an important solution and should also be standardized to attain this purpose.

\section{CONCLUSION}

PACS is likely to be large and complicated because it must process large amounts of data. Var- 

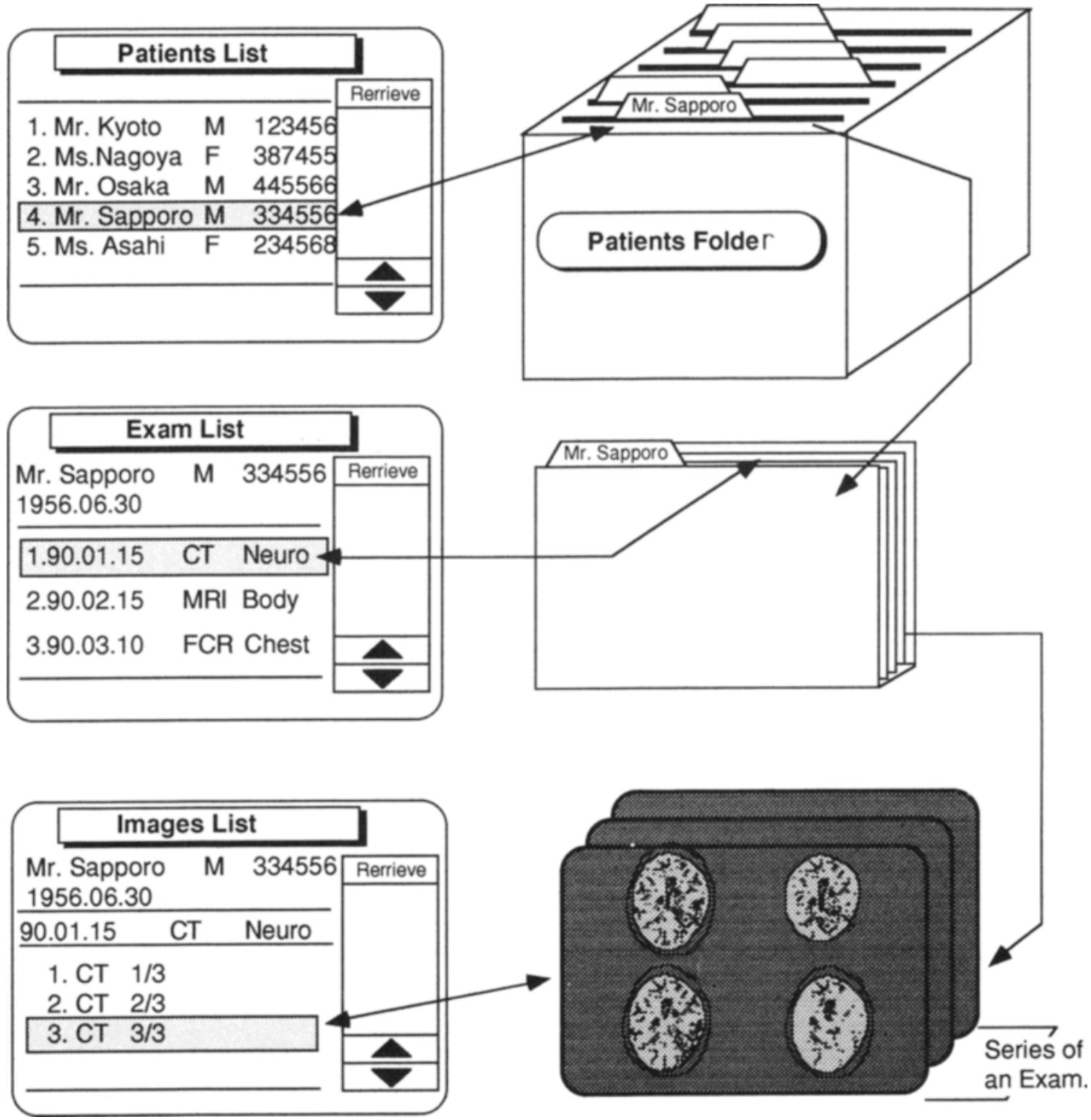

Fig 7. Image retrieval at image displaying terminal.

ious kinds of information generated in the hospital are increasing greatly in quantity, so that they must be effectively controlled and managed. Under these conditions, although many problems remain yet to be resolved by PACS, we can report that PACS and its networks constitute a very important part of the total patient record information system.

\section{REFERENCES}

1. Irie G, Kawakami Y, Kaneda Y, et al: A report on PACS Hokkaido University. Proceedings of the First International conference, IMAC, June 4-8, 1989, IEEE, Washington, DC, pp $72-76$

2. Numbo K, Hirahara T, Takeda I, et al: Features of medium-sized PACS. Med Imaging Technol 7:233-236, 1989

3. Irie G, Kawakami Y, Kaneda Y, et al: A report on PACS at work. Med Imaging Technol 7:227-228, 1989 